\title{
Autophagy and apoptosis in tubular cells following unilateral ureteral obstruction are associated with mitochondrial oxidative stress
}

\author{
YANFANG XU ${ }^{1}$, SHIWEI RUAN ${ }^{3}$, XIAONAN WU ${ }^{4}$, HONG CHEN $^{2}, \mathrm{KE} \mathrm{ZHENG}^{2}$ and BINBIN FU ${ }^{1}$ \\ Departments of ${ }^{1}$ Nephrology and ${ }^{2}$ Pathology, The First Affiliated Hospital, Fujian Medical University, Fuzhou 350005; \\ ${ }^{3}$ Academy of Integrative Medicine, Fujian University of Traditional Chinese Medicine, Fuzhou 350108; \\ ${ }^{4}$ School of Public Health, Fujian Medical University, Fuzhou 350004, P.R. China
}

Received October 3, 2012; Accepted November 30, 2012

DOI: $10.3892 /$ ijmm.2013.1232

\begin{abstract}
Tubular epithelial loss has been shown to be responsible for the formation of atubular glomeruli leading to nephron decomposition and interstitial fibrosis in obstructive uropathy. Cells undergoing apoptosis and autophagic cell death play an important role in this process, yet the mechanisms are not fully understood. In this study, we aimed to investigate whether autophagy cooperating with apoptosis is associated with mitochondrial damage and whether oxidative stress plays an important role in the loss of tubular epithelium following unilateral ureteral obstruction. In this model, we demonstrated that there is coexistence of autophagy and apoptosis with tubular atrophy in obstructed proximal tubules. After unilateral ureteral obstruction (UUO), autophagy in proximal tubular cells was enhanced steadily up to 7 days in the obstructed kidney and declined thereafter, while apoptosis was induced in a timedependent manner from 3 to 14 days. Mitochondrial structure and number also changed during UUO. Lipid peroxidation products, NOX4, and NADPH oxidase activity were also increased in the obstructed renal cortex, and peaked at 7 days. In vitro, we showed that $\mathrm{H}_{2} \mathrm{O}_{2}$ induced mitochondrial injury leading to autophagy and apoptosis through the Beclin 1 pathway and interference with Bcl-2 expression. Thus, our data demonstrate that oxidative stress leading to mitochondrial damage and driven autophagydependent cell death and apoptosis are important mechanisms of tubular decomposition in obstructive nephropathy.
\end{abstract}

\section{Introduction}

Unilateral ureteral obstruction (UUO) in the rodent is the most widely used model of chronic progressive renal disease, quite similar to that developing in many clinical renal disorders (1).

Correspondence to: Dr Yanfang Xu, Department of Nephrology, The First Affiliated Hospital, Fujian Medical University, Chazhong Road 20, Fuzhou 350005, P.R. China

E-mail: xuyanfang99@hotmail.com

Key words: autophagy, apoptosis, obstructive nephropathy, mitochondria
Tubular atrophy and nephron loss is the important pathogenic pathway that leads to progressive reduction in the glomerular filtration rate in chronic renal disease (2). The reduction in proximal tubular mass appears to be the primary determinant of renal parenchymal loss following UUO. In this process, proximal renal tubules undergo oxidant injury, apoptosis and autophagy with widespread mitochondrial injury, which results in tubular collapse and decomposition (3).

Under a low level of injury, autophagy was found to proceed apoptosis and to play a role in cell survival by clearing the degradation of long-lived proteins and subcellular organelles and to re-establish homeostasis (4). When excessive levels of autophagy are activated due to severe injury, it may be detrimental and responsible for non-apoptotic cell death (5). Autophagy induced by UUO has a renoprotective role in the obstructed kidney at an early stage (6). It has recently been reported that under the stimulus of persistent UUO, cytokines and growth factors and oxidative stress may induce proximal tubular epithelial cell death by excessive autophagy and apoptosis (7).

Experimental and clinical evidence indicates that transforming growth factor (TGF)- $\beta 1$ plays a central role in renal fibrosis following obstructive nephropathy. Transgenic overexpression of TGF- $\beta 1$ localized to the proximal tubule induces autophagy and then leads to tubular decomposition, peritubular fibrosis and nephron loss. The process of tubular degeneration in this model was similar in essential features to tubular decomposition following UUO (8). The persistent hypoxia, increasing levels of TGF- $\beta 1$ and other cytokines after UUO, stimulate the overproduction of reactive oxygen species (ROS), which have been previously identified as a key component to mediate autophagy and apoptosis (9). Inhibition of oxidative stress and mitochondrial injury can ameliorate tubular injury and renal interstitial fibrosis in obstructive nephropathy (10). Evidence has shown that selective oxidant injury to the proximal tubules in response to UUO causes widespread DNA damage and mitochondrial injury (11). In this condition, the induction of autophagy may fail to prevent proximal tubule apoptosis, but may contribute to progressive tubular atrophy.

In the present study, we aimed to ascertain whether accumulation of reactive oxygen species induces autophagy and promotes apoptosis, which contribute to renal proximal 
tubular cell death, and represents an important mechanism of tubular decomposition and nephron loss following UUO.

\section{Materials and methods}

Animals and experimental protocols. Sprague-Dawley (SD) male rats (weight, 200-230 g) were subjected to UUO (30 animals) or sham operation (10 animals). All animal experimental procedures were previously approved by the Ethics Committee on Laboratory Animal Research at Fujian Medical University, in accordance with the Guidelines of Experimental Animals of China. The rats were anesthetized by intraperitoneal injection of pentobarbital sodium at a dose of $50 \mathrm{mg} / \mathrm{kg}$. In animals undergoing UUO, the left ureter was ligated with 8-0 nylon, while in the sham-operated mice the ureter was left undisturbed. The rats were sacrificed 3, 7 and 14 days after UUO.

Cell culture. All experiments were performed using renal proximal tubular epithelial cells (NRK-52E) (Chinese Academy of Sciences Shanghai Institute). Cells were maintained in DMEM that contained $4.5 \mathrm{~g} / 1$ glucose (Invitrogen, Carlsbad, CA) supplemented with $5 \%$ fetal bovine serum (FBS), at $37^{\circ} \mathrm{C}$ in $5 \% \mathrm{CO}_{2}$.

Materials and reagents. The antibodies used were as follows: anti-LC3-I and anti-LC3-II (Cell Signaling Technology); antiBeclin 1 (BD Biosciences), $\beta$-actin (Sigma-Aldrich, St. Louis, MO), anti-NOX4 (Abcam), anti-Bax and anti-Bcl-2 (Santa Cruz Biotechnology, Inc., Santa Cruz, CA). The reagents used were as follows: a Trypan Blue Staining Cell Viability Assay, Terminal deoxynucleotidyl transferase-mediated dUTP nick translation end labeling (TUNEL) cell apoptosis detection kit, Hoechst staining kit, and BCA protein assay kit provided by Beyotime Institute of Biotechnology (Haimen, China).

Pathological observation of the kidneys. Kidneys were fixed using $10 \%$ formaldehyde overnight and dehydrated using gradient ethanol. The transparent kidney that was treated with xylene was embedded in paraffin. The pathological changes in the tubulointerstitium were observed using Periodic acid-Schiff (PAS) and Masson's staining. The pathological changes in the tubulointerstitium changes were semi-quantitatively observed using a microscope and scored in a double-blind manner. Ten fields of the PAS-stained slices that did not contain glomeruli were randomly observed (x200). The pathological changes were scored according to the expansion/atrophy of the renal tubules, inflammatory infiltration and interstitial fibrosis. The scores were as follows: normal, 0 ; injured area $<25 \%, 1$; injured area between 25 and 50\%, 2; and injured area $>50 \%, 3$. Collagen deposition in the renal interstitium was semi-quantitatively analyzed using Masson's staining. Twenty renal section fields were randomly selected and observed using a microscope (x200). The semi-quantitative score was obtained by calculating the ratio of the positive collagen-stained area to the area of the entire field. The scores were as follows: positive area $<2 \%$ (normal), 0; positive area between 2 and 10\% (light), 1; positive area between 11 and 20\% (moderate), 2; positive area between 21 and $30 \%$ (severe), 3; and positive area $>30 \%$ (the most severe), 4 .
Electron microscopic (EM) analysis. Autophagosomes were evaluated by electron microscopy. Kidney block samples were fixed in glutaraldehyde and osmium tetroxide, washed with PBS, dehydrated with a series of graded ethyl alcohol solutions. After exchange through acetone, the samples were later embedded in Epon 812. The tissue blocks were made into ultra-thin sections $(70-80 \mathrm{~nm})$ after observation and positioning, and were double stained by uranium and cobalt following standard methods. All of the samples were examined using transmission electron microscopy (TEM) (Philips EM 208).

Detection of SOD, MDA and GSH levels. The kidney was lysed and homogenized, and the homogenate was centrifuged at $4^{\circ} \mathrm{C}$. Levels of superoxide dismutase (SOD), malonaldehyde (MDA) (Jianchen Bioengineering Institute, Najing, China) and glutathione (GSH) (Beyotime Institute of Biotechnology) in the supernatant were detected as per instructions included in the kit.

Isolation of mitochondria from the tissue. All steps were carried out at $4^{\circ} \mathrm{C}$. Mitochondrial isolation buffer (Sigma-Aldrich) was added to the tissue of the renal cortex. The tissue was homogenized and lysated, then subjected to centrifugation at $1,000 \mathrm{xg}$ for $10 \mathrm{~min}$. The supernatants were then centrifuged at $3,000 \mathrm{xg}$ for $10 \mathrm{~min}$. The pellets were resuspended and centrifuged at $1,500 \mathrm{x} g$ for $10 \mathrm{~min}$. The resulting mitochondrial pellet was resuspended and centrifuged at 3,000 and 15,000 $\mathrm{x}$ g and the final light mitochondrial pellet was obtained.

TUNEL staining analysis. Detection of apoptosis in the obstructed kidney was performed following the instructions of the TUNEL assay. Brown granules in the nucleus or cytoplasm of renal tubular epithelial cells represented positive apoptotic cells. Two sections were observed and photographed for each rat. Ten consecutive fields ( $\mathrm{x} 400$ ) were randomly selected, and the nymber of positive cells was assessed using an image analyzer.

Real-time reverse transcription-PCR. Total RNA from the mitochondrial fractions of the obstructed renal cortex was extracted using TRIzol reagent (Gibco-BRL). The sequences of the target gene were obtained from nucleotides of the National Center for Biotechnology Information (NCBI). Primers were synthesized by Sangon Co. (Shanghai, China). The cDNA was obtained using AMV reverse transcriptase. The profile of reverse transcription was as follows: $95^{\circ} \mathrm{C}$ for $15 \mathrm{sec}$, followed by $60^{\circ} \mathrm{C}$ for $1 \mathrm{~min}$. PCR was performed using the following primers: NOX4, 5'-TCAACTGCAGCCTGATCCTTT-3' (sense) and 5'-TCTGTGATCCGCGAAGGTAAG-3' (antisense). The relative expression of NOX4 was obtained as follows: Expression of NOX4/expression of $\beta$-actin.

Immunoblot analyses. The kidney was homogenized in boiling lysis buffer (50 mM Tris, $\mathrm{pH} 7.4,1 \%$ sodium deoxycholate and $0.1 \%$ SDS). Cultured cells were washed with ice-cold PBS and lysed in RIPA lysis buffer. The concentration of proteins was detected by a BCA protein assay kit. Immunoblot analysis was performed in a routine manner.

NADPH oxidase activity. NADPH oxidase activity of mitochondrial fractions in the renal cortex was measured by 


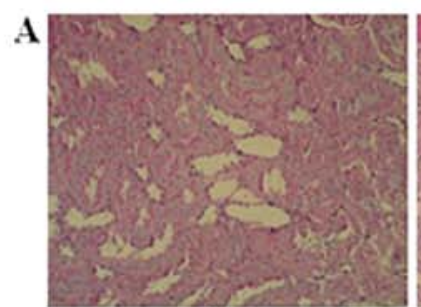

Sham

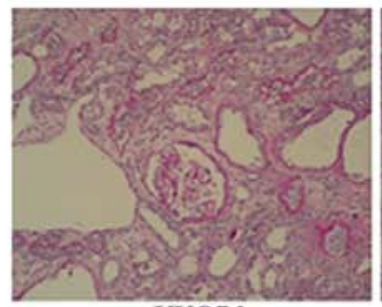

UUO7d

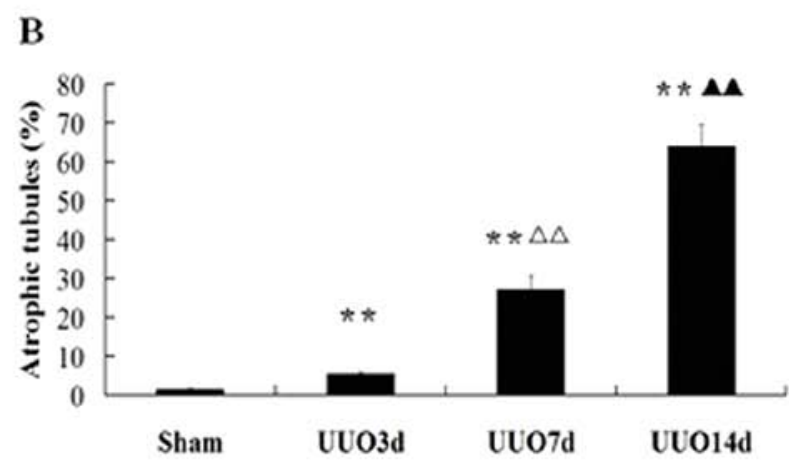

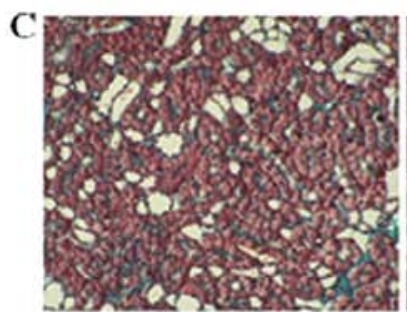

Sham

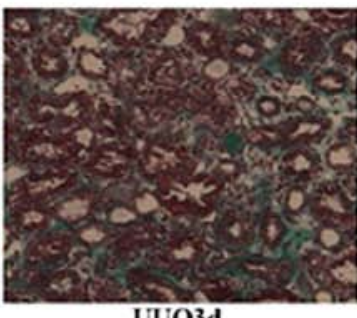

UUO3d

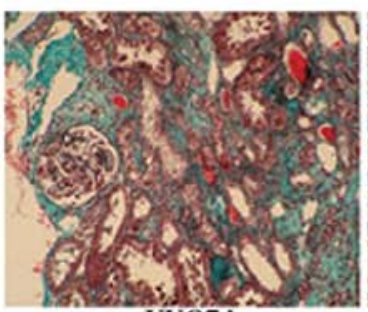

UUO7d

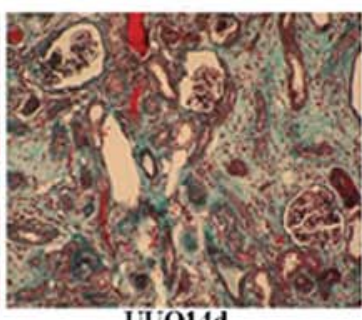

UUO14d

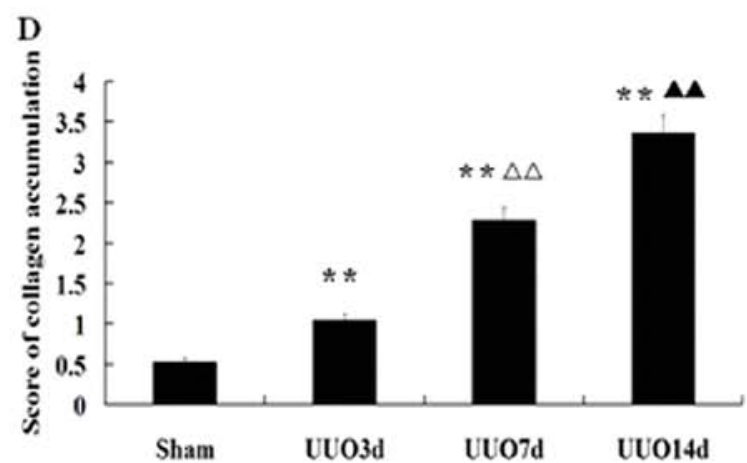

Figure 1. Tubular atrophy and interstitial fibrosis following unilateral ureteral obstruction (UUO). (A) Periodic acid-Schiff staning showed that tubular atrophy was evident after prolonged obstruction. (B) Tubular atrophy was quantified. (C and D) Masson's trichrome staining showed that matrix accumulation increased in a time-dependent manner post UUO and collagen accumulation was examined. (Magnification, $\mathrm{x} 400$ ).

the luminol (5-amino-2,3-dihydro-1,4-phthalazinedione; Sigma-Aldrich) technique. Samples were homogenized and centrifuged. The membrane fraction was lysated and the concentration of proteins was quantified. $\beta$-NADH and luminol in dimethyl sulfoxide were incorporated, and NADPH oxidase activity was evaluated by fluorescence emission. The values were expressed as relative fluorescence units per microgram of protein.

Hoechst 33258 staining. Apoptosis in the cultured cells was evaluated by staining the nuclei with Hoechst staining to determine those with condensed chromatin. Human renal proximal tubular epithelial cells (HRPTEpiCs) were cultured in 6-well flat-bottom plates. After exposure to $0.1 \mathrm{mM} \mathrm{H}_{2} \mathrm{O}_{2}$ for $12 \mathrm{~h}$, cells were fixed, washed with PBS, and then stained with Hoechst $33258(0.5 \mathrm{ml} /$ well $)$ for $5 \mathrm{~min}$. Cells were observed under fluorescence microscopy with a $350 \mathrm{~nm}$ excitation filter and $460 \mathrm{~nm}$ emission filter.

Measurement of mitochondrial membrane potential. To measure mitochondrial depolarization in NRK-52E cells, a JC-1 probe was employed. JC-1 exhibits potential-dependent accumulation in mitochondria, which is indicated by a fluorescence emission shift from 529 to $590 \mathrm{~nm}$. Mitochondrial depolarization is indicated by an increase in the ratio of green:red fluorescence intensity as described previously (12).

Statistical analysis. All experiments were performed in at least triplicate. Data are presented as means \pm the standard deviation (SD) and analyzed using Statistical Product and Service Solutions (SPSS) version 13.0. Between-group differences were analyzed using one-way analysis of variance (ANOVA) and Bonferroni test. $\mathrm{P}<0.05$ was considered as the level of significance.

\section{Results}

Tubular atrophy and interstitial fibrosis following unilateral ureteral obstruction. Periodic acid-Schiff staining was performed to measure tubular atrophy. The proximal tubules were identified by the presence of brush border. In shamoperated kidneys, rare tubular atrophy was observed. Proximal tubular injury was evidenced by the loss of brush border at 3 days, and tubule dilation was commonly noted at 7 days with mild to moderate tubular atrophy after UUO. A minimum of 1,000 tubules were examined for tubular atrophy in each sample. At 14 days, tubular atrophy and wrinkling of the TBM became more predominant (Fig. 1A and B). 

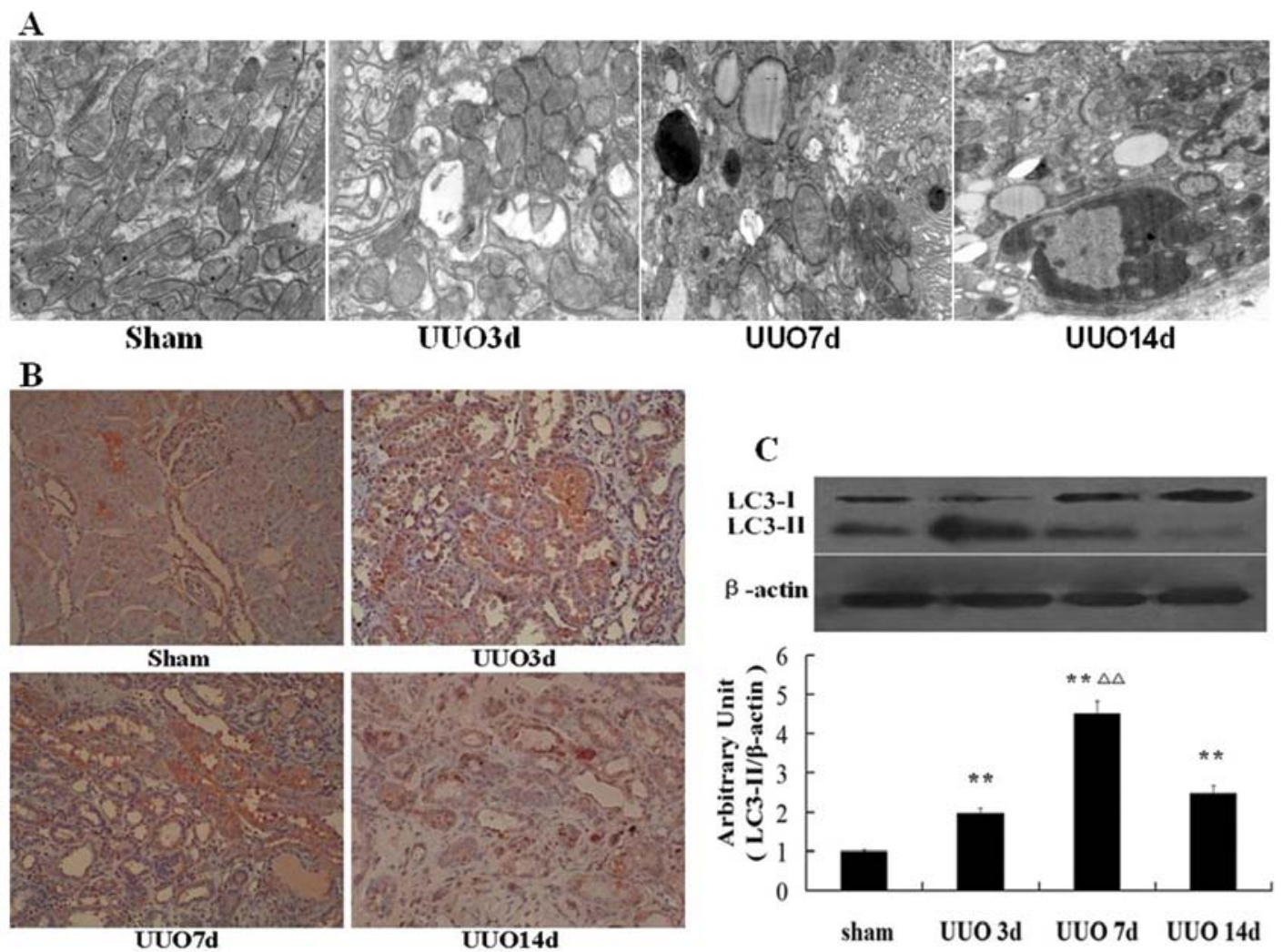

Figure 2. Autophagy was induced in proximal tubules in the obstructed kidney after UUO. (A) Representative transmission electron micrographs. Normal structures of the nucleus, mitochondria, and other cellular organelles were noted in the control kidney, while double membrane vacuoles containing electron dense material and compressed mitochondria with the loss of visible cristae were observed at 3 and 7 days following UUO. Notably, at 14 days after UUO, apoptotic bodies were noted (magnification, x10,000). (B) Light micrographs of immunohistochemical results of LC3 in the cortex. (C) Immunoblot analysis of LC3. $\beta$-actin served as a normalizing control. Values plotted are mean $\pm \mathrm{SD}$ of LC3-II/ $\beta$-actin ratios from three independent experiments. ${ }^{* *} \mathrm{P}<0.01$ compared with sham-operated control group; ${ }^{\triangle} \triangle \mathrm{P}<0.01$ vs. UUO3d.
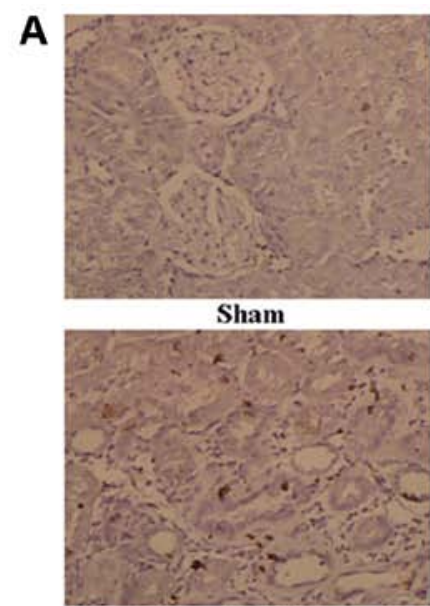

UU07d

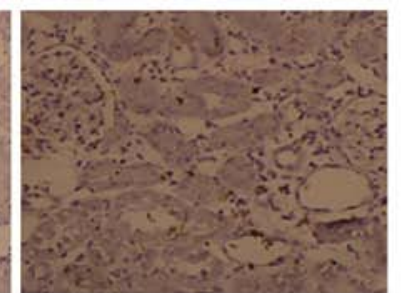

UUO3d

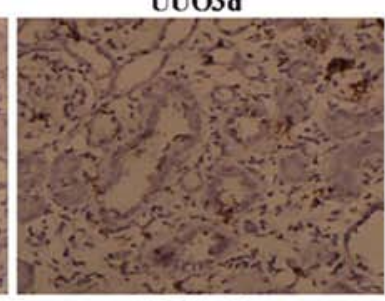

UUO14d

B

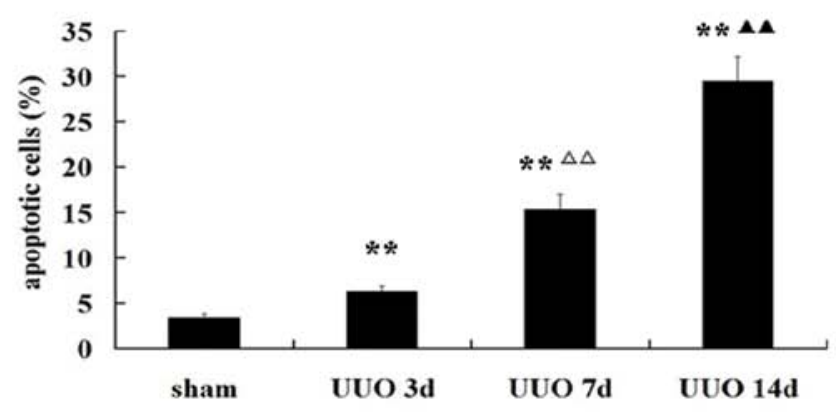

Figure 3. Apoptosis was induced in proximal tubules in the obstructed kidney after UUO. (A) Representative image of TUNEL staining. (B) Quantification of TUNEL-positive cells. ${ }^{\triangle} \mathrm{P}<0.01$ vs. UUO3d; ${ }^{\wedge} \mathrm{P}<0.01$ vs. UUO7d; ${ }^{* *} \mathrm{P}<0.01$ vs. sham-operated control group.

Masson's trichrome staining also showed matrix accumulation and interstitial fibrosis in a time-dependent manner in the obstructed kidney after UUO (Fig. 1C). In comparison to the sham-operated groups, the renal cortex showed higher collagen accumulation in the expanded interstitium after 14 days of UUO (Fig. 1D).
Autophagy was induced in the obstructed renal tubules. Evidence suggests that tubular autophagy follows UUO. To confirm the autophagy of proximal tubular cells, we first examined autophagy by TEM. Rare autophagic vacuoles were detected in the tubular cells of the sham operation rats. However, complete urinary tract obstruction led to an 
Table I. Levels of MDA and antioxidant enzymes in the obstructed kidney.

\begin{tabular}{lllr}
\hline Group & GSH $(\mathrm{g} / \mathrm{g})$ & SOD $(\mathrm{U} / \mathrm{mg})$ & MDA $(\mathrm{nmol} / \mathrm{mg})$ \\
\hline Sham & $65.21 \pm 6.02$ & $502.40 \pm 47.55$ & $8.03 \pm 0.45$ \\
UUO 3d & $51.51 \pm 4.78^{\mathrm{a}}$ & $411.77 \pm 38.13^{\mathrm{a}}$ & $16.19 \pm 1.48^{\mathrm{a}}$ \\
UUO 7d & $37.99 \pm 3.45^{\mathrm{a}, \mathrm{b}}$ & $325.93 \pm 26.12^{\mathrm{a}, \mathrm{b}}$ & $23.09 \pm 2.01^{\mathrm{a}, \mathrm{b}}$ \\
UUO 14d & $31.88 \pm 2.71^{\mathrm{a}, \mathrm{b}}$ & $256.32 \pm 22.79^{\mathrm{a}, \mathrm{c}}$ & $28.59 \pm 2.64^{\mathrm{a}, \mathrm{c}}$ \\
\hline
\end{tabular}

Data are means $\pm \mathrm{SD}, \mathrm{n}=10$ animals in each group. ${ }^{\mathrm{a}} \mathrm{P}<0.01$ vs. sham group; ${ }^{\mathrm{b}} \mathrm{P}<0.01$, vs. UUO $3 \mathrm{~d}$; ${ }^{\mathrm{c}} \mathrm{P}<0.05$, vs. UUO 7d. MDA, malonaldehyde; SOD, superoxide dismutase; GSH, glutathione.

increased level of autophagy (Fig. 2A). Autophagic vacuoles including autophagosomes and autophagolysosomes were markedly increased in the proximal tubular cells at days 3 and 7 after UUO. Double membrane vacuoles containing electron-dense material, degenerating cytoplasmic organelles and cytosol, mitochondria with loss of visible cristae were frequently observed in the tubules. However, these autophagic vacuoles significantly decreased at day 14 after UUO.

To further confirm autophagy, we examined the expression of microtubule-associated protein 1 light-chain 3 (LC3)-II. The conversion of LC3-I to LC3-II is considered a standard marker of autophagy (13). Using immunohistochemical staining, we also demonstrated that the expression of LC3-II to LC3-I increased steadily up to day 7, then declined thereafter (Fig. 2B). In agreement with the immunohistochemical staining, western blotting showed that the contents of LC3-II to LC3-I markedly increased at days 3 and 7, and then decreased towards basal levels at day 14 after UUO (Fig. 2C).

Increased apoptotic cell death following UUO. In the shamoperated kidneys, rare apoptotic tubular cells were observed. In the obstructed tubules, the number of apoptotic cells steadily increased from day 3 to 14 . The number of TUNELpositive cells increased in a time-dependent manner after UUO (Fig. 3). Notably, in Fig. 2A, at 14 days after UUO, apoptotic bodies were also noted. These results indicated the coexistence of autophagy and apoptosis with tubular atrophy in the obstructed proximal tubules. However, autophagy was induced earlier than tubular apoptosis and decreased at day 17 .

Expression of Bcl-2, Bax and Beclin 1 following UUO. Bcl-2 is known to play an anti-apoptotic role in renal tubule damage (14). In this UUO model, immunoblot analysis demonstrated that levels of Bcl-2 protein were modestly increased at day 7 , and markedly decreased at day 14 . However, the proapoptotic (Bax) protein was not significantly upregulated in the obstructed kidney after 14 days. It is known that, Beclin 1 is involved in the initiation of autophagosome formation by forming a multiprotein complex (4). We also confirmed the increased expression of interacting protein Beclin 1 by immunoblot analysis. Beclin 1 protein expression was also significantly increased 7 days after UUO (Fig. 4).

Levels of MDA and antioxidant enzymes in the obstructed kidney. Oxidative stress has been implicated as one of the major underlying mechanisms behind the pathogenesis of
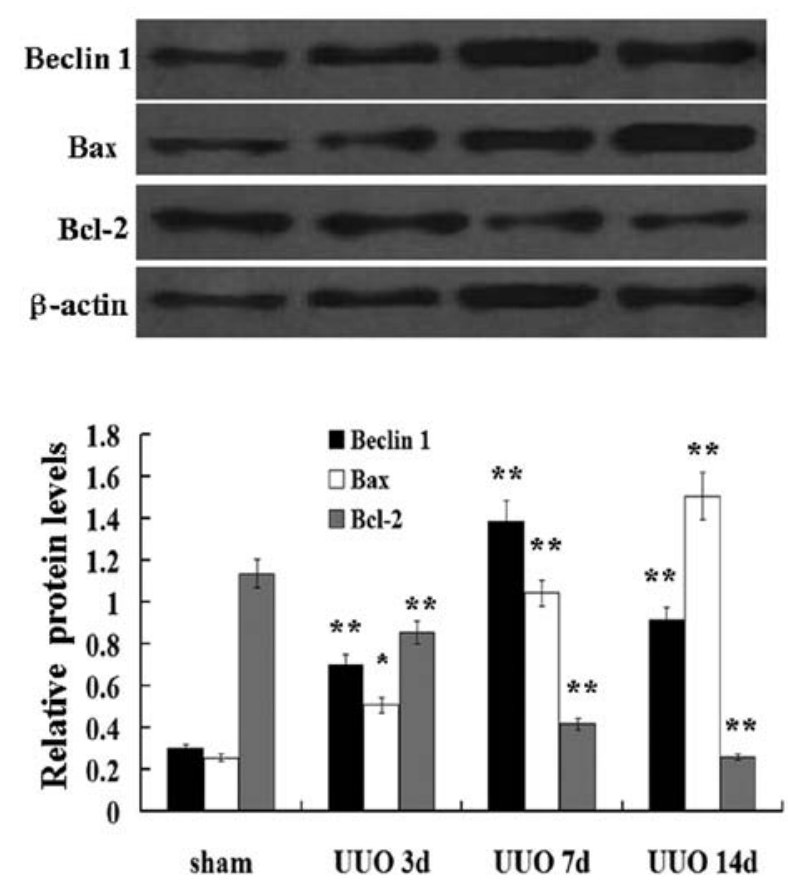

Figure 4. Beclin 1, Bcl-2 and Bax protein expression in renal cortices during UUO. The protein expression was evaluated by western blotting, and protein levels were normalized to $\beta$-actin. ${ }^{* *} \mathrm{P}<0.01$ vs. sham-operated control group.

obstructive uropathy, which has been demonstrated to be related with activation of tubular autophagy and apoptosis (15). MDA is the natural product of lipid peroxidation. The concentration of MDA increases under conditions of oxidative stress. Antioxidant supplementation protects against the progression of renal fibrosis. SOD protects against superoxidemediated cytotoxicity by catalyzing $\mathrm{O}_{2}^{-}$to form $\mathrm{H}_{2} \mathrm{O}_{2}$. The GSH antioxidant system is foremost among the cellular protective mechanisms. Thus, we determined the level of MDA, SOD and GSH in the obstructed kidney. The level of MDA increased while the levels of SOD and GSH decreased in a time-dependent manner in the obstructed kidney (Table I).

NOX4 expression and NADPH oxidase activity in mitochondrial fractions of the renal cortex following UUO. NOX4 is primarily localized in mitochondria by which NOX4 stimulates cell dysfunction and increased cell death (16). The NOX4 mRNA expression was significantly elevated in mitochondrial fractions beginning 3 days after obstruction, 

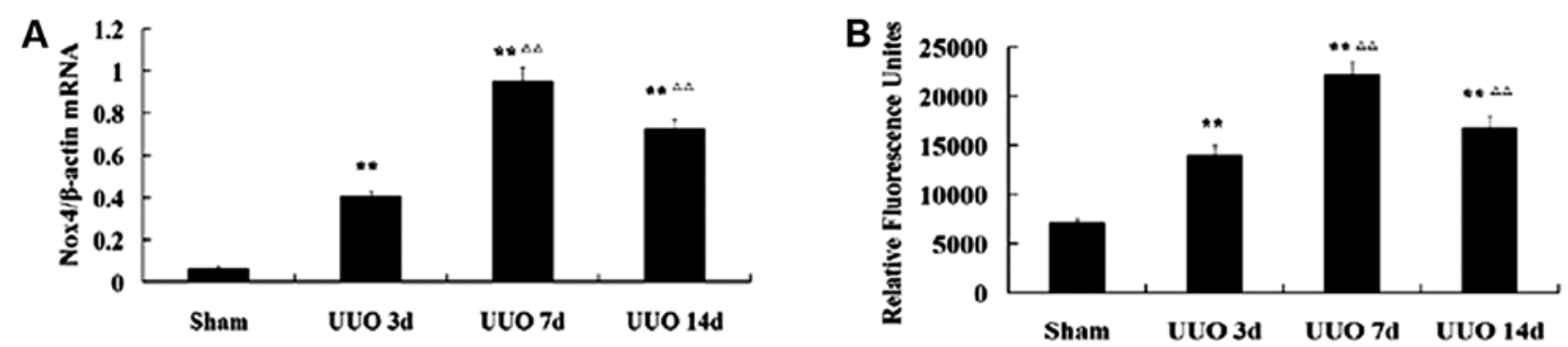

Figure 5. NOX4 expression and NADPH activity in mitochondrial fractions from renal cortices during UUO. (A) Graphical representation of the NOX4/ $\beta$-actin mRNA ratio. (B) NADPH oxidase activity was increased in mitochondrial fractions from obstructed cortices. Data are representative of three independent experiments. ${ }^{* *} \mathrm{P}<0.01$ compared with the sham-operated control group; ${ }^{\triangle \triangle} \mathrm{P}<0.01$ vs. UUO $3 \mathrm{~d}$.
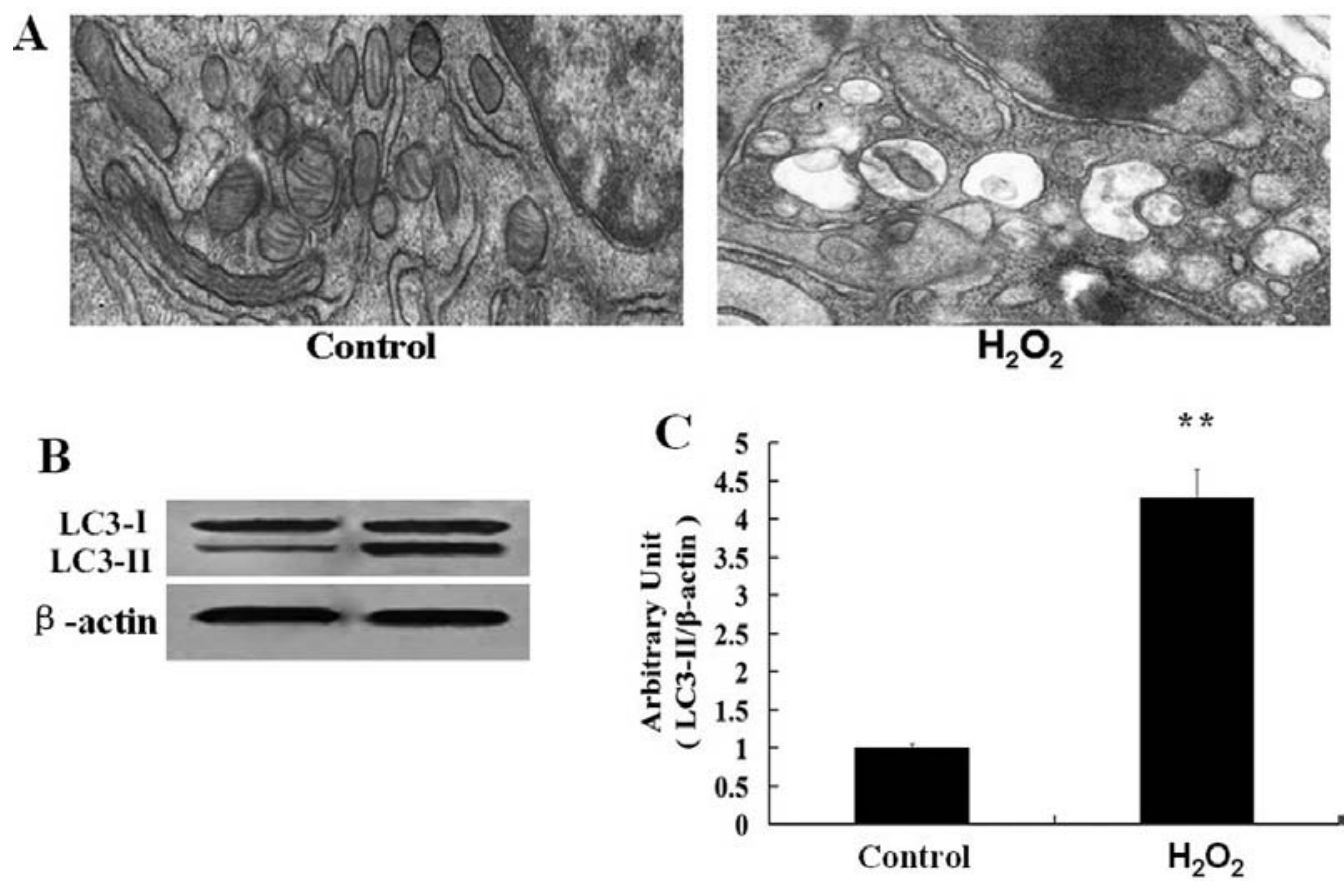

Figure 6. $\mathrm{H}_{2} \mathrm{O}_{2}$ inducs autophagy in NRK-52E cells. NRK-52E cells were incubated in standard medium (control) or medium containing $0.1 \mathrm{mM} \mathrm{H}_{2} \mathrm{O}_{2}$ for $12 \mathrm{~h}$. (A) Ultrastructural changes were visualized by electron microscopy. Abnormal mitochondrial structure, autophagosomes and apoptotic bodies were noted after $\mathrm{H}_{2} \mathrm{O}_{2}$ treatment. (B) The conversion of endogenous LC3-I to LC3-II was visualized using specific antisera on immunoblots and (C) quantified by densitometry. $\beta$-actin served as a normalizing control. Values plotted are the means \pm SD of LC3-II/ $\beta$-actin ratios from three independent experiments. ${ }^{* *} \mathrm{P}<0.01 \mathrm{vs}$. control group.

and reached a peak on day 7. Parallel to this, NADPH oxidase activity was notably enhanced in the renal cortex 7 days after UUO (Fig. 5).

$\mathrm{H}_{2} \mathrm{O}_{2}$ induces parallel apoptosis and autophagy in NRK-52E cells. NRK-52E cells were incubated in the control medium or medium containing $\mathrm{H}_{2} \mathrm{O}_{2}$ for $12 \mathrm{~h}$. We used electron microscopy (EM) to image the autophagic vacuoles in NRK-52E cells. As shown in Fig. 6A, in control cell nuclei and mitochondria were well preserved structures with normal morphology. In contrast, abundant autophagic vacuoles and abnormal mitochondria with dark cristae and condensed matrix were noted in the cells cultured for $12 \mathrm{~h}$ with $0.1 \mathrm{mM} \mathrm{H}_{2} \mathrm{O}_{2}$. Notably, some cells showed nuclei with condensed chromatin or fragmented apoptotic bodies under TEM (Fig. 6A). The conversion of LC3-I to LC3-II by lipd phosphatidylethanolamine (PE) conjugation in autophagy during autophagosome formation is one of the estabished steps (4). Western blot analysis indicated that the conversion of LC3-I to LC3-II increased after exposure to $\mathrm{H}_{2} \mathrm{O}_{2}$ for $12 \mathrm{~h}$ (Fig. $6 \mathrm{~B}$ and $\mathrm{C}$ ).

The percentage of apoptotic cells was visualized by Hoechst staining. Compared with the control, the number of positive cells markedly increased after exposed to $\mathrm{H}_{2} \mathrm{O}_{2}$ (Fig. 7A and B). Reduction in the mitochondrial membrane potential $(\Delta \Psi \mathrm{m})$ and increase in mitochondrial membrane permeability correlated with apoptosis. Red fluorescence indicates cells with a stable $\Delta \Psi \mathrm{m}$, while green fluorescence indicates cells with a low $\Delta \Psi \mathrm{m}$. When NRK-52E cells were exposed to $\mathrm{H}_{2} \mathrm{O}_{2}$ for $12 \mathrm{~h}$, the relative ratio of green fluorescence increased compared with the untreated control cells, indicating increased number of cells undergoing apoptosis (Fig. 7C and D).

As a well-known anti-apoptotic gene, Bcl-2 is involved in the regulation of autophagy and apoptosis. Evidence shows that Bcl-2 inhibits Beclin 1-dependent autophagy by inter- 
A

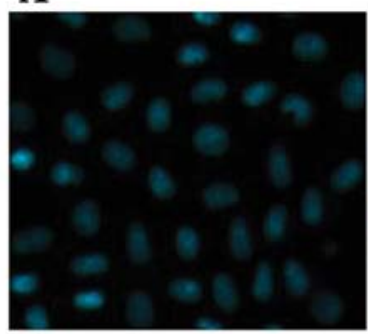

Control
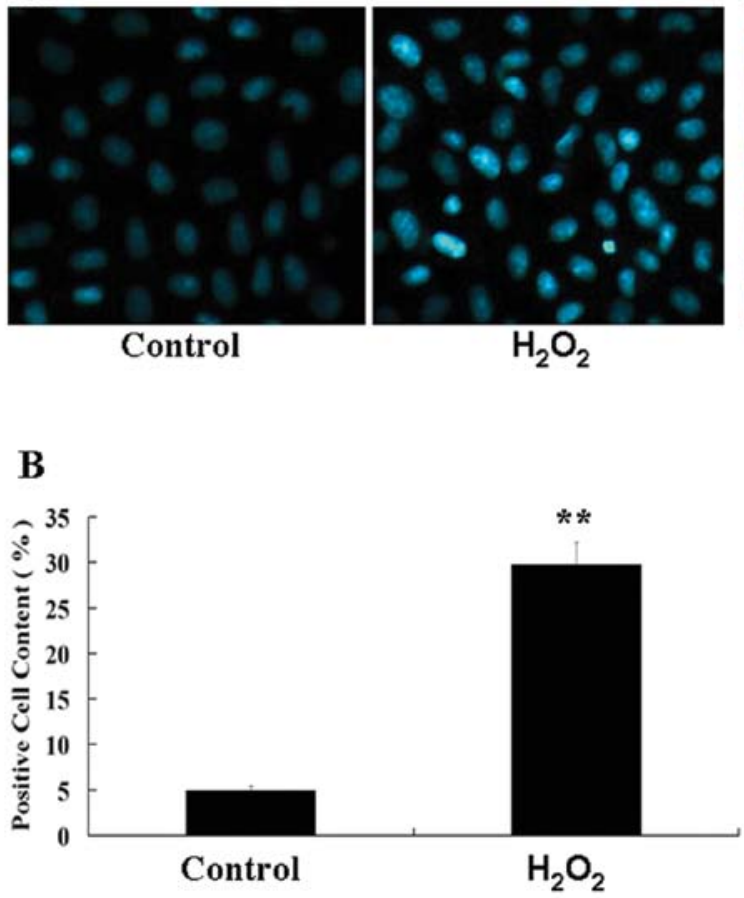

$\mathrm{H}_{2} \mathrm{O}_{2}$
C

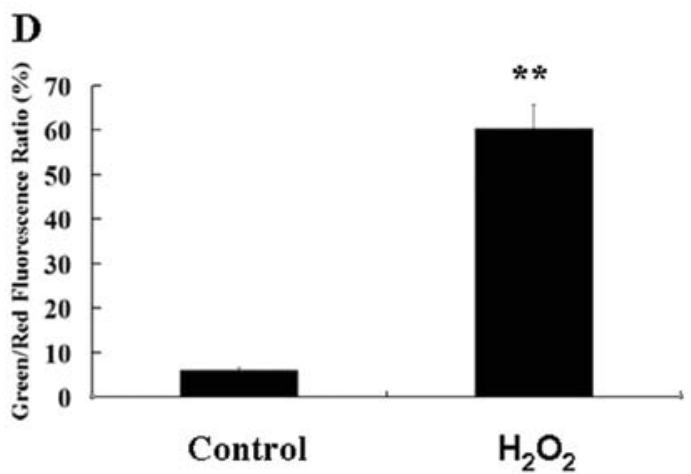

Figure 7. $\mathrm{H}_{2} \mathrm{O}_{2}$ induces apoptosis in NRK-52E cells. Cells were incubated as described in Fig. 5. (A) Cells were stained with Hoechst staining to visualize nuclei and (B) the percentage of apoptotic versus viable cells was determined based on chromatin condensation, which indicates cells undergoing apoptosis. (C) Cells were stained with JC-1 and imaged by fluorescence microscopy (magnification, x400). (D) The ratio of green to red fluorescence intensity was quantified to determine a reduction in mitochondrial membrane potential, a marker of early apoptosis. All values are means \pm SD from 10 independent micrographs from each group. ${ }^{* *} \mathrm{P}<0.01$ vs. control group.

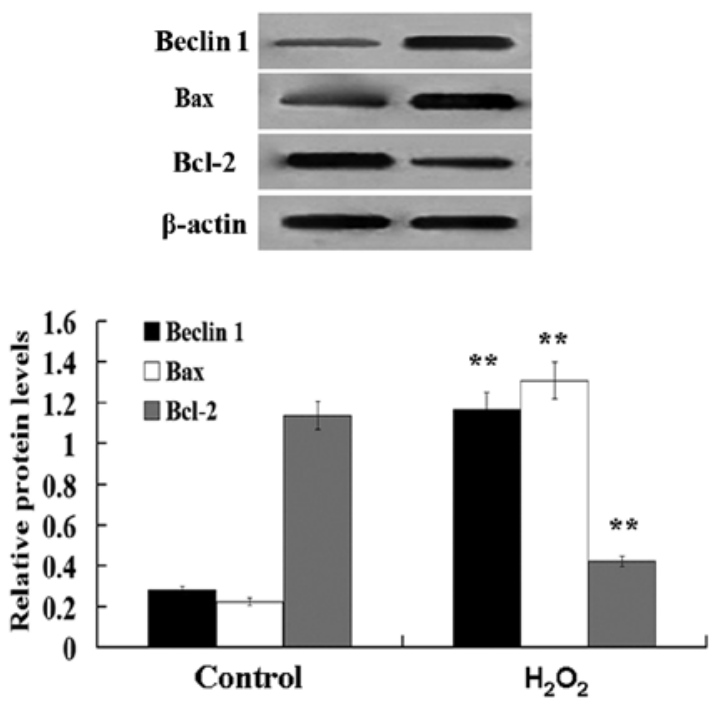

Figure 8. Beclin, Bcl-2 and Bax protein expression in NRK-52E cells cultured with $\mathrm{H}_{2} \mathrm{O}_{2}$. The protein expression was evaluated by western blotting, and protein levels were normalized to $\beta$-actin. ${ }^{* *} \mathrm{P}<0.01$ vs. control group.

acting with Beclin 1. Overexpression of Bcl-2 significantly inhibited $\mathrm{H}_{2} \mathrm{O}_{2}$-induced formation of autophagic vacuoles and suppressed the mitochondrial fragmentation (13). Immunoblot analysis demonstrated that $\mathrm{H}_{2} \mathrm{O}_{2}$ downregulated anti-apoptotic (Bcl-2) and stimulated pro-apoptotic (Bax) proteins, and also increased Beclin 1 protein expression (Fig. 8).

\section{Discussion}

Surgical UUO in a variety of experimental animals has been used as a model of progressive chronic kidney. Previous studies have shown that epithelial-mesenchymal transition (EMT) plays an important role in nephron loss and interstitial fibrosis obstructive uropathy (1). Recent evidence suggests another pathway for functional nephron loss in UUO, which is proximal tubular injury and rapid formation of atubular glomeruli (17). The formation of atubular glomeruli proceeded to total decomposition of tubular cells as a result of autophagy and apoptosis. It is well known that apoptosis is increased in obstructed renal tubules (18). However, others recently reported that autophagy contributes to excessive cell death and tubular atrophy is another potential mechanism of nephron loss in obstructive uropathy (7). Autophagy can also act as a mechanism of cell survival, but it can lead to cell death in response to persistent stress (19). Under the stimulus of persistent UUO, the induction of autophagy fails to prevent progressive destruction of the proximal tubular mass (7). Transgenic overexpression of TGF- $\beta 1$ localized to the proximal tubule also induces extensive autophagy, and the autophagocytic process culminates in total disconnection of cells from the TBM and tubular decomposition. In the present study, we showed that autophagy and apoptosis proceeded by proximal tubule injury contributed to tubular atrophy and renal fibrosis (8). However, autophagy increased within the first week and then declined, while apoptosis continued to increase through 2 weeks. Thus, the induction of autophagy 
in proximal tubules failed to prevent apoptosis, but promoted apoptosis in this UUO model.

Nephron loss in UUO has been associated with increased free radical reactions and localized oxidant damage in proximal tubules $(10,18)$. Mitochondria are the major source of ROS. NOX4 is the major NADPH oxidase isoform expressed in renal cells and is primarily localized in mitochondria. It plays an important role in mediating ROS generation $(10,20)$. In this study, NOX4 expression increased significantly in mitochondrial fractions of the obstructed renal cortex during UUO. The mitochondria-rich proximal tubules appeared to be the most susceptible to obstructive injury. Electron microscopy showed that mitochondria were of an increased size and contained dilated cristae and large spaces in their interiors after UUO. The increased ROS may also be due to impaired antioxidant capacity, such as an increased level of MDA and reduced activity of SOD and GSH of the obstructed renal cortex. Thus, oxidative stress in the renal cortex increased in response to UUO.

Mitochondrial oxidative stress may contribute to the decline in mitochondrial functions associated with the collapse of mitochondrial membrane potential and further transient increase in ROS generation. These events may result in autophagy, apoptosis or necrosis (21). Autophagy is one of the defense mechanisms protecting cells against oxidative stress. However, persistent enhanced oxidative stress can induced parallel apoptotic cell death and autophagic cell death (22). In this condition, autophagy may cooperate with the apoptotic machinery by acting upstream of apoptosis, converging with the apoptotic pathway. In this UUO model, autophagy occurred earlier than apoptosis, but it did not inhibit apoptosis. Thus, autophagy and apoptosis are coordinated processes which contribute to tubular atrophy.

Oxidative stress plays an important role in various renal pathological conditions. ROS are generated at high levels to induce oxidation and damage DNA, mitochondria, endoplasmic reticulum and other organelles or macromolecules $(13,23)$. Reactive oxygen species (ROS) have been shown to mediate cellular responses including EMT or mitochondriadependent apoptosis and autophagy in various types cells $(13,24)$. Previously, it was observed that ROS mediated TGF- $\beta 1$-induced autophagy, which promoted apoptosis in renal tubular epithelial cells (19). Rasbach and Schnellmann (25) showed that exposure to oxidants decreased mitochondrial function and biogenesis, which was associated with autophagy in RPTCs (25). In the present study, we also demonstrated that hydrogen peroxide $\left(\mathrm{H}_{2} \mathrm{O}_{2}\right)$ induced parallel autophagy and mitochondria dysfunction-mediated apoptosis. However, it is still unclear whether $\mathrm{H}_{2} \mathrm{O}_{2}$-induced autophagy contributes to apoptosis in renal tubular epithelial cells.

It has recently been shown that Bcl-2 family proteins, key modulators of apoptosis, represent a point of convergence of the autophagic and apoptotic processes. The anti-apoptotic proteins, such as Bcl-2, Bcl-xL and Bcl-B have been shown to inhibit autophagy by binding to Beclin 1 , a critical protein involved in initiation of autophagosome formation $(13,26,27)$. In this UUO model, we showed that pro-apoptotic Bcl-2 family protein Bax increased while anti-apoptotic protein Bcl-2 decreased in a time-dependent manner. Notably, at day 7 , Beclin 1 was significantly enhanced. These results indicate that downregulation of Bcl-2 decreased its capacity to interact with Beclin 1 and failed to suppress autophagic cell death. Due to oxidative stress, the mitochondrial dysfunction by accumulation of intracellular ROS has been associated with autophagy and apoptosis (13). As a well-known anti-apoptotic gene, overexpression of Bcl-2 stabilized mitochondria, blocked the classic apoptotic pathways, affected binding with Beclin 1 and inhibited autophagy $(13,28)$. In vitro, our results showed that $\mathrm{H}_{2} \mathrm{O}_{2}$ treatment increased the expression of Beclin 1 protein and decreased the expression of Bcl-2, and also induced mitochondria dysfunction and autophagy. However, under the condition of oxidative stress, the insight into the interplay between apoptosis and autophagy needs further investigation.

In summary, we demonstrated that both autophagy and apoptosis occurred in the tubular epithelial cells following obstructive uropathy, associated with oxidative stress. Under the stimulus of persistent UUO, the induction of autophagy in the proximal tubule did not play a protective mechanism, but contributed to cell death. Further understanding of the mechanism of the relationship between autophagy and apoptosis of tubular epithelial cells in obstructive uropathy may facilitate the development of therapeutic strategies to prevent end-stage renal disease.

\section{Acknowledgements}

This study was supported in part by the Nature Science Foundation from Fujian Provincial Department of Science and Technology (no. 2011J01159), in part by an innovatory research fund (no. 2011-CX-10) from the Department of Health of Fujian Province, and partly by a Key Research Fund JA12136 from Education Department of Fujian Province of China.

\section{References}

1. Yang JW and Liu YH: Blockage of tubular epithelial to myofibroblast transition by hepatocyte growth factor prevents renal interstitial fibrosis. J Am Soc Nephrol 13: 96-107, 2002.

2. Zeisberg $M$ and Neilson EG: Mechanisms of tubulointerstitial fibrosis. J Am Soc Nephrol 21: 1819-1834, 2010.

3. Forbes MS, Thornhill BA and Chevalier RL: Proximal tubular injury and rapid formation of atubular glomeruli in mice with unilateral ureteral obstruction: a new look at an old model. Am J Physiol Renal Physiol 301: F110-F117, 2011.

4. Nishida K, Yamaguchi O and Otsu K: Crosstalk between autophagy and apoptosis in heart disease. Circ Res 103: 343-351, 2008.

5. Luo S and Rubinsztein DC: Apoptosis blocks Beclin 1-dependent autophagosome synthesis: an effect rescued by Bcl-xL. Cell Death Differ 17: 268-277, 2010.

6. Kim WY, Nam SA, Song HC, et al: The role of autophagy in unilateral ureteral obstruction rat model. Nephrology 17 : 148-159, 2012.

7. Li L, Zepeda-Orozco D, Black R and Lin FM: Autophagy is a component of epithelial cell fate in obstructive uropathy. Am $\mathbf{J}$ Pathol 176: 1767-1778, 2010.

8. Koesters R, Kaissling B, Lehir M, et al: Tubular overexpression of transforming growth factor-betal induces autophagy and fibrosis but not mesenchymal transition of renal epithelial cells. Am J Pathol 177: 632-643, 2010.

9. Ferraro E and Cecconi F: Autophagic and apoptotic response to stress signals in mammalian cells. Arch Biochem Biophys 462: 210-219, 2007.

10. García IM, Altamirano L, Mazzei L, et al: Role of mitochondria in paricalcitol-mediated cytoprotection during obstructive nephropathy. Am J Physiol Renal Physiol 302: F1595-F1605, 2012. 
11. Forbes MS, Thornhill BA, Minor JJ, Gordon KA, Galarreta CI, and Chevalier RL: Fight-or-flight: murine unilateral ureteral obstruction causes extensive proximal tubular degeneration, collecting duct dilatation, and minimal fibrosis. Am J Physiol Renal Physiol 303: F120-F129, 2012.

12. $\mathrm{Xu} \mathrm{YF,} \mathrm{Ruan} \mathrm{SW,} \mathrm{Xie} \mathrm{H} \mathrm{and} \mathrm{Lin} \mathrm{JM:} \mathrm{Role} \mathrm{of} \mathrm{LOX-1} \mathrm{in}$ Ang II-induced oxidative functional damage in renal tubular epithelial cells. Int J Mol Med 26: 679-690, 2010.

13. Zhang H, Kong X, Kang J, Su J, Li Y, Zhong JT and Sun LK: Oxidative stress induces parallel autophagy and mitochondria dysfunction in human glioma U251 cells. Toxicol Sci 110: 376-388, 2009

14. Miyajima A, Chen J, Lawrence C, et al: Antibody to transforming growth factor-beta ameliorates tubular apoptosis in unilateral ureteral obstruction. Kidney Int 58: 2301-2313, 2000.

15. Docherty NG, O'Sullivan OE, Healy DA, Fitzpatrick JM and Watson RWG: Evidence that inhibition of tubular cell apoptosis protects against renal damage and development of fibrosis following ureteric obstruction. Am J Physiol Renal Physiol 290: F4-F13, 2006.

16. Ago T, Kuroda J, Pain J, Fu C, Li H and Sadoshima J: Upregulation of Nox4 by hypertrophic stimuli promotes apoptosis and mitochondrial dysfunction in cardiac myocytes. Circ Res 106: 1253-1264, 2010.

17. Chevalier RL and Forbes MS: Generation and evolution of atubular glomeruli in the progression of renal disorders. J Am Soc Nephrol 19: 197-206, 2008.

18. Chevalier RL: Pathogenesis of renal injury in obstructive uropathy. Curr Opin Pediatr 18: 153-160, 2006.

19. Xu YF, Yang SY, Huang JY, Ruan SW, Zheng Z and Lin JM: TGF- $\beta 1$ induced autophagy and promoted apoptosis in renal tubular epithelial cells. Int J Mol Med 29: 781-790, 2012.
20. Kuroda J, Ago T, Matsushima SJ, Zhai PY, Schneiderb MD and Sadoshim J: NADPH oxidase 4 (Nox4) is a major source of oxidative stress in the failing heart. Proc Natl Acad Sci USA 107: 15565-15570, 2010.

21. Martinet W and De Meyer GR: Autophagy in atherosclerosis: a cell survival and death phenomenon with therapeutic potential. Circ Res 104: 304-317, 2009.

22. Addabbo F, Montagnani M and Goligorsky MS: Mitochondria and reactive oxygen species. Hypertension 53: 885-892, 2009.

23. Periyasamy-Thandavan S, Jiang M, Wei Q, Smith R, Yin XM and Dong Z: Autophagy is cytoprotective during cisplatin injury of renal proximal tubular cells. Kidney Int 74: 631-640, 2008.

24. Rhyu DY, Yang YQ, Ha H, Lee GT, Song JS, Uh ST and Lee HB: Role of reactive oxygen species in TGF-betal-induced mitogenactivated protein kinase activation and epithelial-mesenchymal transition in renal tubular epithelial cells. J Am Soc Nephrol 16: 667-675, 2005.

25. Rasbach KA and Schnellmann RG: Signaling of mitochondrial biogenesis following oxidant injury. J Biol Chem 25: 2355-2362, 2007.

26. Robert G, Gastaldi C, Puissant A, et al: The anti-apoptotic Bcl-B protein inhibits BECN1-dependent autophagic cell death. Autophagy 8: 1-13, 2012.

27. Oberstein A, Jeffrey PD and Shi Y: Crystal structure of the Bcl-XL-Beclin 1 peptide complex: Beclin 1 is a novel BH3-only protein. J Biol Chem 282: 13123-13132, 2007.

28. Pattingre S, Tassa A, Qu X, Garuti R, Liang XH, Mizushima N, et al: Bcl-2 antiapoptotic proteins inhibit Beclin 1-dependent autophagy. Cell 122: 927-939, 2005. 